\title{
PROPOSAL OF CRITERION WEIGHTING THEORY ${ }^{1}$
}

\author{
Soushi Suzuki \\ Graduate School of Engineering, Hokkai-Gakuen University \\ South 26 West 11 Chuo-ku, Sapporo 064-0926 Japan \\ s-suzuki@ss20.eng.hokai-s-u.ac.jp
}

\begin{abstract}
In the absolute measurement approach (AMA) of analytic hierarchy process (AHP) some absolute criterion are set, and they are weighting through evaluation by the examinees. Each alternative is evaluated by its weight. In comparison with a relative measurement approach (RMA), this is less burden some for the examinee. However, the process of the weighting of absolute criterion still presents burdens for the examinee. Thus, we constructed a weighting theory of criterion from the viewpoint of Psychophysics, and set semantic weight of each criterion from this theory. In this study we propose an evaluation method of the semantic measurement approach that can reduce burden to the examinee.
\end{abstract}

\section{Introduction}

Generally, accuracy and practicability are required of an evaluation method. Saaty (1986) proposed AMA in AHP. This method sets absolute criterions which are weighted by the evaluation made by examinees. Then, each alternative for each criteria is evaluated based on this result. The process of the weighting of absolute criterion considerably burdens the examinees. The author focussed on absolute criterion. A weighting theory of criterion was developed from the viewpoint of Psychophysics. In addition, the author proposes a new method in which the practicability of evaluation is improved.

\section{Criterion Weighting Theory}

In AMA, semantic stimulation by adjective is considered to be the same among people of the same culture. It is considered that an adjective, for example "excellent," gives a stimulation which is similar for someone of the same culture. In attempting to establish a theory for determining a value for semantic stimulation, we focused on a Psychophysics study by Weber (1860). In his study on discrimination threshold, he proposed Weber's law (1).

$$
\frac{\Delta I}{I}=\text { const }
$$

where, $I$ is the stimulation strength, and $\Delta I$ is the discrimination threshold at the stimulation strength. If we assume that Weber's law is satisfied by an increase in the weight of criterion that corresponds to the increase in stimulation strength, then (2) can be derived.

$$
d y=k \frac{d z}{z}
$$

where, $\mathrm{Y}$ is the stimulation of an adjective that expresses criterion, $\mathrm{Z}$ is the weight of criterion, dy is increase in the stimulation of the adjective, $\mathrm{dz}$ is increase in the weight of criterion, and $\mathrm{K}$ is a proportional constant.

\footnotetext{
'Acknowledgement: The author wishes to express deep thanks to Professor Hideo Igarashi, Dr.eng, of Hokkai-Gakuen University, for his generous guidance.
} 
By integrating both sides of (2),

$$
z=\alpha \exp (\beta y)
$$

where $\alpha$ is discrimination threshold of the criterion weight, and $\beta$ is a parameter.

Equation 3 expresses what we call "Criterion Weighting Theory (CWT)".

\section{The Suitability of CWT}

A questionnaire survey by pairwise comparison between each criterion was carried out in order to measure the actual weight of each criterion. Because this research method of determining criterion weight by pairwise comparison is an extraction criterion of the process of pairwise comparison among criterion in AMA, it is appropriate. In general evaluation can be made to the opposing adjectives pairs (e.g., "good vs. bad") that are most frequently used. We chore: 1 . terrible, 2. bad, 3. average, 4. good, 5. excellent. Questionnaire respondents were 58 university students (male: 55 , female: 3 ). Valid respondents $($ C.I. $<0.10)$ numbered 44 . Table-1 shows pairwise comparison of the results.

Table -1 Results of Pairwise Comparison

\begin{tabular}{|l|c|c|c|}
\hline & mean value & variance & standard deviation \\
\hline 1.terrible & 0.03282 & 0.00001 & 0.00300 \\
\hline 2.bad & 0.06392 & 0.00005 & 0.00731 \\
\hline 3.average & 0.13047 & 0.00018 & 0.01334 \\
\hline 4.good & 0.26080 & 0.00032 & 0.01796 \\
\hline 5.excellent & 0.51199 & 0.00059 & 0.02420 \\
\hline
\end{tabular}

Based on the questionnaire results, table-1 shows the parameters that gives minimum sums of squares of residual values for 1 to 5 .

The result is:

$$
\begin{aligned}
& \dot{z}=0.0163 \exp (0.6901 y) \\
& \mathrm{R}^{2}=0.9999 \quad \text { (coefficient of correlation) }
\end{aligned}
$$

(4) is the solid line in Figure-1.

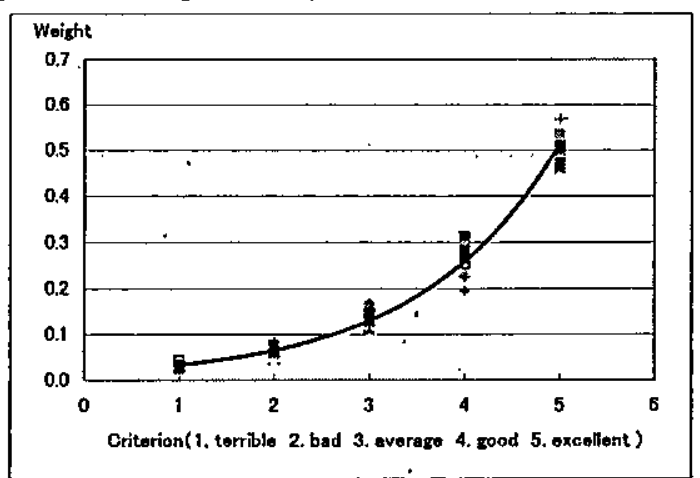

Figure -1 Criterion Weight Theory

From Figure-1, it is clear that the theoretical values and actual values agree well. The weights of the theoretical values were: 'terrible: 0.033 ', 'bad: 0.065 ', 'average: 0.129 ', 'good: 0.258 ', 'excellent: 0.514 '.

\section{Semantic Measurement Approach}

On the basis of CWT, new evaluation method that semantically improves AMA and that maintains AMA's advantages is proposed. It is described as follows: "The weight of each criterion is set by the common weight calculated based on CWT, and all examinees evaluate all alternatives according to this weight." The author has named this new method, "Semantic Measurement Approach (SMA)". It offers considerable advantages:

1. It is possible to considerably reduce the burden on the examinee, because pairwise comparison between criterions is not required.

2. Continuous evaluation between adjectives becomes possible, as CWT is able to express the evaluation between two adjectives.

3. Advantage \#2 means that estimated results can be expressed visually and linguistically.

\section{Conclusion}

This study proposed a new theory, that estimates the weight for adjectives of criterion. Theoretical value was found to agree well with actual value for "good vs. bad ". In addition, SMA was proposed on the basis of this result. Future study is needed for the cases of criterions such as non-opposite adjective pairs or 3-4 stages. 